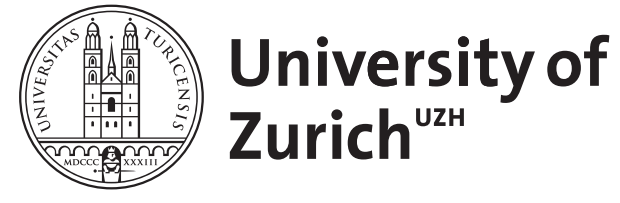

\title{
Abschiedseditorial für die JLM
}

von Eckardstein, Arnold

DOI: https://doi.org/10.1515/jlm.2006.056

Posted at the Zurich Open Repository and Archive, University of Zurich ZORA URL: https://doi.org/10.5167/uzh-154353

Journal Article

Published Version

Originally published at:

von Eckardstein, Arnold (2006). Abschiedseditorial für die JLM. Journal of Laboratory Medicine, 30(6):363.

DOI: https://doi.org/10.1515/jlm.2006.056 


\section{Editorial}

\section{Abschiedseditorial für die JLM}

Die vorliegende Ausgabe der LaboratoriumsMedizin ist die Letzte, die unter meiner Schriftleitung entstanden ist. Mit Beginn des nächsten Jahres werden Herr Prof. Matthias Nauck aus Greifswald und Herr PD Dr. Michael Steiner aus Rostock die Schriftleitung der LaboratoriumsMedizin übernehmen.

In den vergangen 7 Jahren meiner Schriftleitung hat der publizierende Verlag gewechselt und sich das Titelbild der LaboratoriumsMedizin mehrmals gewandelt. Dennoch blieb das inhaltliche Konzept konstant, nämlich die Publikation von schwerpunkmässig zusammengefassten Übersichtsartikeln zum Zwecke der Fortbildung (Continous Medical Education). Dank des grossen Einsatzes der mich unterstützenden Fachredakteure, die Autoren für unser Konzept zu begeistern verstanden, gelang es in den vergangenen Jahren, die 6 Ausgaben der Laboratoriumsmedizin mit 40-50 Artikeln pro Jahr zu füllen (Abbildung $1 \mathrm{~A}$ und $1 \mathrm{~B}$ ). Aus den vielen persönlichen Ansprachen habe ich entnommen, dass dieses Konzept auch bei Ihnen als Leser auf grosse Zustimmung stösst. Leider ist es den beiden Verlagen und mir in zwei Anläufen nicht gelungen, auch Medline und Thompson Scientific zu überzeugen, die Zeitschrift in ihre Listen aufzunehmen. Trotzdem halte ich es nach wie vor für wichtig, dass Labormediziner und Klinische Chemiker oder Klinische Biologen im deutschsprachigen Raum ein Forum haben, das innen erlaubt, mit den rasanten Fortschritten und dem immensen Wissensgewinn unserer Faches Schritt zu halten. Ich hoffe deswegen sehr, dass Sie meinen Nachfolgern nicht nur als Leser die Treue halten, sondern sich auch als Autoren zur Verfügung stellen, um die Gemeinschaft der Labormediziner von Ihrem Wissen profitieren zu lassen.

Schließlich möchte ich mich bei vielen Menschen bedanken, ohne deren Engagement es mir nicht möglich gewesen wäre, mein Konzept für die LaboratoriumsMedizin umzusetzen: es sind dies die Fachredakteure, die sich über Jahre hinweg um die Akquisition von Autoren und Artikeln bemüht haben, ein Geschäft, dass leider nicht immer sehr einfach ist. Es sind dies Frau Dr. Williamson und Frau Hand, die mir in Münster bzw. in Zürich bei der Editierung der Manuskripte hilfreich zur Seite standen. Zunächst der Blackwell Verlag und seit 2004 der Walter de Gruyter Verlag bzw. die dort jeweils für unsere Zeitschrift zuständigen Mitarbeiterinnen, Frau Addicks bzw. Frau Jahnke waren in all den Jahren sehr hilfreich und vor allem geduldig und nachsichtig mit mir, wenn ich die Fristen sehr strapazierte. Schließlich möchte ich mich beim Vorstand der Deutschen Gesellschaft für Laboratoriumsmedizin bzw. der Deutschen Vereinten Gesell- schaft für Klinische Chemie und Laboratoriumsmedizin für die kontinuierliche Unterstützung und Ermutigung in machmal nicht einfachen Zeiten bedanken.

A Umfang der JLM in den Jahren 1997-2006

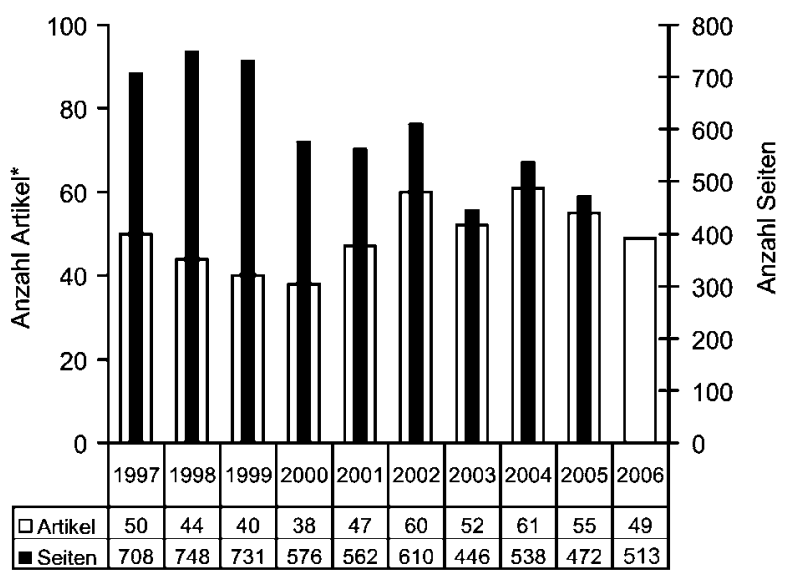

*: Nur reguläre Artikel (keine Mitteilungen abstracts, editorials usw.)

B Formen der Artikel

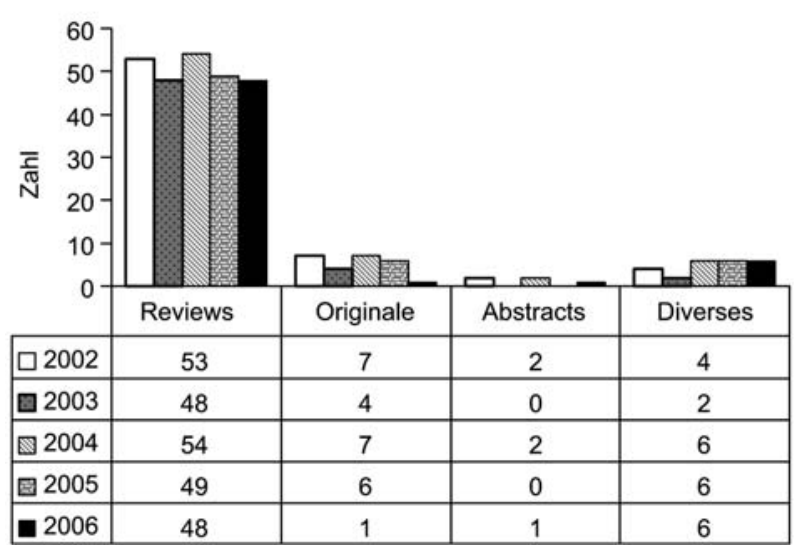

Prof. A. von Eckardstein Universitäts-Spital Zürich Institut für Klinische Chemie Rämistrasse 100

8091 Zürich

Tel.: $+44 / 2552260$

Fax: $+44 / 2554590$

E-mail: arnold.voneckardstein@usz.ch 